\title{
DIAGNÓSTICO CLÍNICO E ANATOMOPATOLÓGICO: DISCORDÂNCIAS
}

\author{
José Roberto Alves, Milton Hida, Gisele Alborghetti Nai* \\ Trabalho realizado na Faculdade de Medicina da Universidade do Oeste Paulista - \\ UNOESTE, Presidente Prudente, SP.
}

RESUMO - Muitas patologias na prática clínica geram discordâncias quanto a sua identificação, não somente pela sua semelhança com outras lesões mas também pela sua semântica.

OBJEtivo. Este trabalho visa esclarecer quais discordâncias são mais freqüentes na prática clínica, fornecer novos conhecimentos para facilitar a identificação das patologias de maiores controvérsias e ampliar seus diagnósticos diferenciais.

Métodos. Revisamos 1.825 laudos de biópsias referentes ao período de 1992 até 1999, pertencentes ao Laboratório de Anatomia Patológica da Unoeste, sendo excluídos 439 laudos que não apresentavam hipótese diagnóstica ou que tinham como hipótese sinais e sintomas clínicos ou "a esclarecer". Confrontamos a hipótese clínica com o diagnóstico anatomopatológico, obtendo-se 444 $(32,05 \%)$ casos discordantes.
Resultados. Observamos que as maiores discordâncias foram entre o diagnóstico clínico de hanseníase, que em $65,7 \%$ dos casos tratavam-se de dermatites crônicas inespecíficas, entre cisto sebáceo, que em $80 \%$ dos casos tratavam-se de cisto de inclusão epidérmica e aborto incompleto, que em $68,2 \%$ dos casos tratavam-se de aborto completo.

Conclusão. Concluímos que uma adequada definição de conceitos, uma anamnese criteriosa e estreita correlação das características clínicas das lesões promovem um menor número de discordâncias entre os diagnósticos clínico e anatomopatológico.

Unitermos. Discordâncias. Controvérsias. Diagnóstico clínico anatomopatológico.

\section{INTRODUÇÃO}

Muitas patologias na prática clínica geram discordâncias quanto a sua identificação, não somente pela sua semelhança com outras lesões mas também pela sua semântica, gerando diagnósticos clínicos e anatomopatológico divergentes.

Para avaliarmos tais discordâncias, suas possíveis causas e quais os maiores índices de erro, revisamos 1.825 casos de biópsias de nosso serviço no período de 1992 a 1999.

\section{Métodos}

Foram revisados, consecutivamente e retrospectivamente, 1.825 laudos de biópsias de pacientes referentes ao período de 1992 até 1999, enviados ao laboratório de Anatomia Patológica de nossa instituição pelos médicos da região de Presidente Prudente-SP.

Foram excluídos 439 laudos de biópsias, que não apresentaram hipótese diagnóstica (HD) ou tinham como hipótese

*Correspondência

Rua José Bongiovani, 700 - Cidade Universitária CEP: 19050-900 - Presidente Prudente - SP sinais e sintomas clínicos ou ainda o diagnóstico "a esclarecer", totalizando 1.386 laudos de biópsias avaliados.

Os dados colhidos através dos laudos catalogados no Laboratório Anatomopatológico - UNOESTE foram: sexo, idade, material recebido, hipótese clínica e diagnóstico anatomopatológico (DAP).

\section{Resultados}

Dos 1.825 laudos de biópsia revisados, 439 laudos de biópsias $(24,11 \%)$ foram excluídos, destes 281 (15,40\%) foram anulados pois continham como HD sintomas ou sinais ou procedimentos técnicos (Ex.: menorragia, vasectomia) ou HD "a esclarecer", e I58 (8,7|\%) que não continham HD. Assim, analisamos 1.385 laudos de biópsias. Destes, 444 (32,05\%) mostraram discordâncias entre HD e DAP.

As principais HDs discordantes foram as seguintes, em ordem decrescente de freqüência: cisto sebáceo, hanseníase, abortamento incompleto, nevus pigmentados, carcinoma basocelular (Tabela I).

Dessas HDs, freqüentemente divergentes, verificamos que os cistos sebáceos em quase todos os casos foram discordantes
( $94,57 \%$ do total de casos), sendo a maioria diagnosticada anatomopatologicamente como cisto de inclusão epidérmica $(75,70 \%$ do total de casos) (Tabela 2).

Em relação aos 43 casos de hanseníase, houve discordância em $81,39 \%$, sendo que a maioria teve o diagnóstico anatomopatológico de dermatite crônica inespecífica (65,7l\% dos casos) (Tabela 3).

Nos I I 5 casos com HD de abortamento incompleto existiu divergência de 19,13\% do total de casos sendo que $68,18 \%$ tratavam-se de abortamentos completos, pois o material enviado tratava-se de placenta inteira com variadas alterações (Tabela 4).

Com relação aos 86 casos HD de nevo pigmentado, 24,42\% destes foram discordantes, tratando-se a maioria dos casos discordantes de ceratose seborréica (4,65\% dos casos) ao exame anatomopatológico (Tabela 4).

Dos 56 casos com HD de carcinoma basocelular, 35,68\% destes foram discordantes, sendo que o exame anatomopatológico mostrou tratar-se de carcinoma espinocelular em $40 \%$ dos casos discordantes e ceratose actínica em 20\% destes (Tabela 5). 


\begin{tabular}{lcc}
\hline \multicolumn{3}{c}{ Tabela I - HDs que mais freqüentemente apresentaram discordâncias } \\
com relação ao diagnóstico anatomopatológico
\end{tabular}

Tabela 2 - Diagnóstico anatomopatológico discordante dos casos com HD de cisto sebáceo

\begin{tabular}{lcc}
\hline \multicolumn{1}{c}{$\begin{array}{c}\text { Diagnóstico } \\
\text { anatomopatológico }\end{array}$} & $\begin{array}{c}\mathbf{N}^{0} \text { de } \\
\text { Casos }\end{array}$ & $\%$ \\
\cline { 2 - 3 } Cisto Epidérmico & 28 & 75,67 \\
Carcinoma Basocelular & $\mid$ & 2,70 \\
Cisto Pilonidal & $\mid$ & 2,70 \\
Cisto Simples Benigno & $\mid$ & 2,70 \\
DermatiteAguda & $\mid$ & 2,70 \\
Dermatite Crónica & $\mid$ & 2,70 \\
Fibrose Dérmica & $\mid$ & 2,70 \\
Lipoma & $\mid$ & 2,70 \\
Total & 35 & 94,57 \\
\hline
\end{tabular}

* Casos de cisto sebáceo concordantes: dois casos (5,40\%).

\begin{tabular}{|c|c|c|}
\hline $\begin{array}{c}\text { Diagnóstico } \\
\text { anatomopatológico }\end{array}$ & $\begin{array}{l}N^{0} \text { de } \\
\text { Casos }\end{array}$ & $\%$ \\
\hline $\begin{array}{l}\text { Dermatite crônicainespećfica } \\
\text { Ceratose seborréica } \\
\text { Fibrose dérmica } \\
\text { Hiperceratose } \\
\text { Compatível com molusco contagioso } \\
\text { Compatível com papiloma virus } \\
\text { Epitélio sem atipias } \\
\text { Lentigo Simples } \\
\text { Psoríase }\end{array}$ & $\begin{array}{c}23 \\
3 \\
2 \\
2 \\
1 \\
1 \\
1 \\
1 \\
1\end{array}$ & $\begin{array}{r}53,49 \\
6,98 \\
4,65 \\
4,65 \\
2,32 \\
2,32 \\
2,32 \\
2,32 \\
2,32\end{array}$ \\
\hline Total & 35 & 81,37 \\
\hline
\end{tabular}

* Casos de Hanseníase concordantes: oito casos (气18,60\%)

\section{Discussão}

Dos 1.386 laudos de biópsias analisados, 32,05\% evidenciaram a discordância entre diagnóstico clínico e anatomopatológico.

As lesões de pele foram as que mais resultaram em diagnósticos discordantes, tendo quatro representantes das cinco patologias com maiores discordâncias verificadas nos laudos de biópsias.

Dessas, o cisto sebáceo apresentou quase todos os casos com discordância (pouco mais de $94,5 \%$ ). Acredita-se que a principal justificativa para tal fato deva-se a problemas semânticos, sendo assim é válido esclarecer alguns conceitos:

a) Cisto sebáceo era um termo usado para cistos pilares (ou cistos triquilemais ou cistos pilosos)2;

b) Atualmente os principais cistos cutâneos são: os cistos epidérmicos (ou de inclusão epidérmica) e os cistos pilares $2,5,12,13,15$; c) O cisto pilar é clinicamente indiferenciável do cisto epidérmico, no entanto, ele é de natureza mais firme que o cisto epidérmico ${ }^{12,13}$;

d) Cistos epidérmicos são os cistos cutâneos mais freqüentes e ocorrem principalmente na face (nariz) e tronco superior. Já os cistos pilares são os menos freqüentes entre os cistos cutâneos e estão localizados principalmente no couro cabeludo $(90 \% \text { dos casos })^{12}$.

No entanto, é necessário ressaltar que as lesões císticas da pele (cistos pilonidal, epidérmico e simples benigno) e lipoma fazem parte do diagnóstico diferencial dos cistos pilares (sebáceos), juntamente com o esteatocistoma multiplex $^{12,13}$. Assim, casos plenamente discordantes (não pertencentes ao diagnóstico diferencial de cistos pilares) totalizaram II,43\% destes.

Com relação à hanseníase (Moléstia de Hansen) que foi a segunda patologia com o maior número de casos discordantes (81,37\% do total de casos), acredita-se que a maior justificativa foi a solicitação de biópsias pelo simples fato de o paciente ser contactante para hanseníase e ter uma lesão cutânea polimorfa aparentemente típica desta patologia. Assim, para diminuir a incidência de tais casos discordantes, é valido esclarecer alguns pontos sobre a variabilidade de apresentações clínicas da moléstia de Hansen conforme o estado

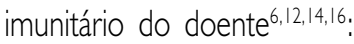

a) Hanseníase indeterminada ocorrerá quando o paciente for imunocompetente (plena capacidade imunológica para responder aos antígenos do Mycobacterium leprae) e se apresentará como manchas hipocrômicas ou eritêmato-hipocrômicas, única ou em pequeno número, com hipoestesia, na maioria das vezes não delimitadas, localizadas na face, superfície extensora dos membros ou no tronco (principalmente nas nádegas), podendo ser acompanhada de hipo ou anidrose, assim como queda de pelos no local6, 14 ;

b) Hanseníase tuberculóide, no caso também de paciente imunocompetente, se apresentará como lesões eritêmato-acastanhadas polimórficas tipo pápula ou placas delimitadas (cheias ou com elevação apenas das bordas), com ou sem hipocromia central, de tamanho e número variados, acompanhadas de perda evidente da sensibilidade e distúrbios na sudorese; 
Tabela 4 - Diagnóstico anatomopatológico discordante para os casos com HD de abortamento incompleto

\begin{tabular}{|c|c|c|}
\hline $\begin{array}{c}\text { Diagnóstico } \\
\text { anatomopatológico }\end{array}$ & $\begin{array}{l}N^{0} \text { de } \\
\text { Casos }\end{array}$ & $\%$ \\
\hline $\begin{array}{l}\text { Placenta de } I^{0} \text { Trimestre de Gestação } \\
\text { acompanhada de variadas alteraç̧ôes }\end{array}$ & 15 & 13,04 \\
\hline $\begin{array}{l}\text { Presença de fragmentos de endométrio } \\
\text { decidualizado }\end{array}$ & 5 & 4,35 \\
\hline Endométrio de padrão secretor tardio & । & 0,87 \\
\hline Mola hidatiforme parcial & | & 0,87 \\
\hline Total & 22 & 19,13 \\
\hline
\end{tabular}

* Casos de Abortamento Incompleto concordantes: 93 casos (80,87 \%)

Tabela 5 - Diagnóstico anatomopatológico discordante para os casos com HD de nevus pigmentado

\begin{tabular}{|c|c|c|}
\hline $\begin{array}{c}\text { Diagnóstico } \\
\text { anatomopatológico }\end{array}$ & $\begin{array}{l}\mathrm{N}^{0} \text { de } \\
\text { Casos }\end{array}$ & $\%$ \\
\hline Ceratose Seborréica & 4 & 4,65 \\
\hline Carcinoma Basocelular & 2 & 2,32 \\
\hline Fibroma & 2 & 2,32 \\
\hline Hiperceratose & 2 & 2,32 \\
\hline Hiperplasia Sebácea & 2 & 2,32 \\
\hline Fibrose Dérmica & l & 1,16 \\
\hline NevoSpitz & I & 1,16 \\
\hline Dermatite Liquenóide & I & 1,16 \\
\hline HiperplasiaAdenomatosa & 1 & 1,16 \\
\hline Papulose Bowenoide & I & 1,16 \\
\hline Dermatite Crônica & I & 1,16 \\
\hline Trombo Oclusivo Intradérmico & l & 1,16 \\
\hline Verruga Vulgar & i & 1,16 \\
\hline Nevo Lipomatoso & I & 1,16 \\
\hline Total & 21 & 24,37 \\
\hline
\end{tabular}

* Casos de Nevus Pigmentado concordantes: 65 casos (75,58 \%)

Tabela 6 - Diagnóstico anatomopatológico discordante para os casos com HD de carcinoma basocelular

\begin{tabular}{|c|c|c|}
\hline $\begin{array}{c}\text { Diagnóstico } \\
\text { anatomopatológico }\end{array}$ & $\begin{array}{l}N^{\circ} \text { de } \\
\text { Casos }\end{array}$ & $\%$ \\
\hline $\begin{array}{l}\text { Carcinoma Espinocelular } \\
\text { CeratoseActínica } \\
\text { Ceratose Seborréica } \\
\text { Tricoadenoma } \\
\text { Edema e Hemorragia Cutânea } \\
\text { Cisto de Inclusão Epidérmica } \\
\text { Melanoma Maligno } \\
\text { Elastose Solar }\end{array}$ & $\begin{array}{l}8 \\
4 \\
3 \\
1 \\
1 \\
1 \\
1 \\
1\end{array}$ & $\begin{array}{l}14,28 \\
7,14 \\
5,36 \\
1,78 \\
1,78 \\
1,78 \\
1,78 \\
1,78\end{array}$ \\
\hline Total & 20 & 35,68 \\
\hline
\end{tabular}

* Casos de carcinoma basocelular concordantes: 36 casos (64,28 \%).

c) Hanseníase virchowiana ocorre em pacientes imunocomprometidos (imunidade celular deficiente, deprimida) que apresentarão lesões simétricas, polimorfas, de coloração ferruginosa tipo pápulas, tubérculos, nódulo, placas, ulcerações e infiltração difusa cutânea, não delimitadas, acompanhados de hipoestesia e distúrbios de sudorese e pilosos, que podem ocorrer por todo o corpo ${ }^{6,14}$; d) Hanseníase dimorfa ocorre quando o paciente está com imunidade não plenamente competente ou deprimida, sendo uma forma caracterizada clinicamente entre a forma tuberculóide e virchowiana. No entanto, os casos típicos podem se apresentar como placas eritematosas com limites mal definidos, com limites internos nítidos e externos mal definidos, localizadas em todos os segmentos corporais, sendo geralmente numerosas, acompanhadas de alterações de sensibilidade ${ }^{6,14}$.

Além disso, devemos lembrar que não existe outra doença infecto-contagiosa que apresente lesões com distúrbios cutâneos de sensibilidade ${ }^{6}$.

Em suma, pode-se compor algumas regras que facilitarão a formulação do diagnóstico definitivo de hanseníase, baseados nas apresentações clínicas dermatológicas ${ }^{14}$ :

a) Tudo que parecer lesão de hanseníase indeterminada não é se for normo-sensível e histamino-completa, exceto no caso do nevo anêmico;

b) Tudo que parecer lesão de hanseníase tuberculóide, mas que não seja rigorosamente anestésica, não será lesão característica desta;

c) Tudo que parecer lesão de hanseníase virchowiana, cuja baciloscopia para bacilo álcool-ácido-resistente (BAAR) não seja evidentemente positiva, não será lesão característica desta, sendo que a pesquisa da sensibilidade pouco auxiliará neste caso, pois poderá existir pouca ou nenhuma perda da sensibilidade.

Com base nas características supracitadas, tem-se que antes de se pedir alguma biópsia para possível paciente com hanseníase devese lembrar que ele, além de indivíduo contactante, deverá ter distúrbios cutâneos, principalmente de sensibilidade (hipo ou anestesia) e de sudorese (hipo ou anidrose). Além disso, devemos ressaltar que todos os diagnósticos clínicos discordantes (todos não pertencentes ao diagnóstico diferencial da hanseníase) evidenciados em nossa pesquisa poderiam ser evitados se se levasse em conta a hipo ou anestesia das lesões cutâneas.

Com relação aos casos discordantes de abortamento incompleto $(19,13 \%$ do total de casos), novamente o fator semântica esteve 
presente, basicamente relacionado ao mecanismo pelo qual qual se produz o abortamento e ao conteúdo expulso via vaginal. Para termos um abortamento incompleto será necessário que alguma parte do concepto (anexos embrionários) ou da placenta não sejam expulsos na sua totalidade, pois se forem teríamos um abortamento completo $0^{4,9}$.

Com relação aos $24,4 \%$ de casos discordantes de nevus pigmentados (ou melanocíticos), acreditamos que a principal justificativa seja a caracterização dermatológica da lesão erroneamente sobre alguns aspectos principais, isto porque essa é uma lesão bem delimitada, pigmentada, com distribuição uniforme de seu pigmento e não crostosa, na maioria dos $\operatorname{casos}^{12}$, ou o fato de se chamar de nevus a qualquer mal formação do tecido cutâneo.

Ainda, em relação aos casos de nevus pigmentado, devemos comentar que a maioria (86,36\% dos casos discordantes) das HDs discordantes verificadas em nosso estudo não eram hipóteses pertencentes ao diagnóstico diferencial desta dermatose ${ }^{12}$.

A maioria dos casos discordantes de carcinoma basocelular (CBC) (85\% destes) pertencia ao diagnóstico diferencial para esta dermatose. Para minimizar a incidência de casos discordantes, gostaríamos de levantar alguns pontos, principalmente, com relação ao carcinoma espinocelular (CEC), o principal diagnóstico anatomopatológico nos casos discordantes (40\%). Apesar de constar do diagnóstico diferencial do CBC, poderiam ser evitadas tais discordâncias se atentássemos para algumas particularidades dessa dermatose:

a) $\mathrm{O} C B C$ é o câncer da pele mais comum (71\% a $81 \%$ dos cânceres cutâneos) $)^{15}$;

b) Raramente ocorre metástase: 0,0028\% dos casos ${ }^{15}$;

c) O CBC ocorre nos dois terços superiores da face (acima da linha que passa pelos lóbulos das orelhas e comissuras labiais), tronco e extremidades, nunca ocorrendo em palmas, plantas e mucosas $^{12}$;

d) O CEC ocorre no terço inferior da face, dorso das mãos, mucosa bucal e genitália externa ${ }^{12}$.

\section{Conclusão}

Uma anamnese mais criteriosa, além de uma estreita correlação entre características clínicas (idade, sexo, história e exame físico), definição adequada de conceitos e domínio dos diagnósticos diferenciais de cada patologia são fatores extremamente importantes para se melhorar a qualidade do diagnóstico clínico e aproximá-lo do diagnóstico anatomopatológico.

\section{Conflito de interesse: não há.}

\section{SUMMARY}

\section{Clinical Diagnosis and Anatomic- Pathologic Diagnosis: Disagreements}

Many pathologic entities in the clinical practice generate disagreements regarding its identification, not only by its likeness with other lesions but also by its semantics.

BACKGROUND. The goal ofthis workisto clarify which disagreements are more frequent in the clinical practice, supply new knowledges to facilitate the identification of the larger pathologies controversies and to enlarge its differentials diagnosis.

Methods: We revised 1825 reports of referringbiopsies to the period of / 992 up to 1999 , belonging to the Laboratory of Pathological Anatomy of Unoeste, being excluded 439 lauds that did not introduce diagnostic hypothesis, or that had as signals hypothesis and clinical indications or "for clearing". We confronted the clinical hypothesis with the diagnosis Anatomicpathological, obtaining itself 444 (32.05\%) of discordant cases.

RESULTS. Note that the biggest disagreements were between Hansen's disease clinical diagnosis, which in $65.7 \%$ cases were chronicles Unspecific Dermatitises, between Sebaceous Cyst, which in $80 \%$ cases were cyst of Epidermical Inclusion and Incomplete Abortion, which in $68.2 \%$ cases were complete Abortion.

CONCLUSION. We conclude that an adequate concepts definition discerning an anamnese and narrow correlation of the clinical characteristics of the lesions promote a minor disagreements number among clinical diagnosis and Anatomicpathological. [Rev Assoc Med Bras 2004; 50(2): |78-8I]

KEY wORDS: Disagreements. Controversies. Diagnosis, clinical. Anatomic-pathologic.

\section{REFERÊNCIAS}

I. Anderson WAD, Kissane JM. Patologia. $7^{\mathrm{a}}$ ed. Rio de Janeiro: Guanabara Koogan; 1982.

2. Arnold HL, Odom RB, James WD. Doença de pele de Andrews. Dermatologia clínica. 8a ed. São Paulo: Malone LTDA; 1994.

3. Barreto ML, Carmo EH. Tendências recentes das doenças crônicas no Brasil. In: Lessa I. O adulto brasileiro e as doenças da modernidade. Epidemiologia das doenças crônicas não-transmissíveis. São Paulo: Hucitec/Abrasco; 1998. Cap. I.

4. Bastos AC. Noções de ginecologia. $9^{a}$ ed. São Paulo: Atheneu; 1994.

5. Bork K, Bräuninger W. Dermatologia clínica: diagnóstico e terapia. $2^{\mathrm{a}}$ ed. São Paulo: Manole; 1998.

6. Ghidella C. Hanseníase. Casio Ghidella Personal Home Page's 2000 Ago [cited 2003 Jan I0] Available from: http://www.geocities. com/ hanseniase.html.

7. Kalache A, Veras RP, Ramos LR. O envelhecimento da população mundial. Um desafio novo. Rev Saúde Pública 1987; 21:200-10.

8. Murphy GF, Elder DE. Atlas of tumor pathology. Washington (DC): Armed Forces Institute of Pathology; 1991. p.278.

9. Rezende J, Montenegro CAB. Obstetrícia fundamental. $8^{a}$ ed. Rio de Janeiro: Guanabara Koogan; 2000.

10. Robbins SL, Citran RS, Kumar V. Robbins patologia estrutural e funcional. $4^{\mathrm{a}} \mathrm{ed}$. Rio de Janeiro: Guanabara Koogan; 1991.

II. Rouquayrol MZ, Almeida Filho N. Epidemiologia e saúde. $5^{a}$ ed. Rio de Janeiro: Medsi; 1999.

12. Sampaio SAP, Rivitti EA. Dermatologia. $2^{\text {a }}$ ed. São Paulo: Artes Médicas; 2001.

13. Sampaio SAP. Dermatologia básica. $3^{\mathrm{a}}$ ed. São Paulo: Artes Médicas; 1989.

14. Schechter M, Marangoni DV. Doenças Infecciosas: conduta diagnóstica e terapêutica. $2^{\text {a }}$ ed. Rio de Janeiro: Guanabara Koogan; 1998.

15. Sittart JAS, Pires MC. Dermatologia para o clínico. São Paulo: Editora Lemos Editorial e Gráficos; 1997.

16. Veronesi R, Focaccia R. Veronesi: tratado de infectologia. $2^{\text {a }}$ reimpressão. São Paulo: Atheneu; 1999. p. 1000.

17. Wyngaarden JB, Smith LH. Cecil tratado de medicina interna. $18^{\mathrm{a}}$ ed. Rio de Janeiro: Guanabara Koogan; 1990.

Artigo recebido: 25/04/03

Aceito para publicação: |8/06/03 